\title{
Motor disorders of the oesophagus in gastro-oesophageal reflux
}

\author{
M J MAHONY, ${ }^{*}$ M MIGLIAVACCA, ${ }^{*}$ L SPITZ, $\dagger$ AND P J MILLA* \\ Departments of ${ }^{*}$ Child Health and †Paediatric Surgery, Institute of Child Health and Hospital for Sick \\ Children, Great Ormond Street, London
}

SUMMARY Mechanisms of gastro-oesophageal reflux were studied by oesophageal manometry and $\mathrm{pH}$ monitoring in 33 children: nine controls, 15 with gastro-oesophageal reflux alone, and nine with reflux oesophagitis. A total of 122 episodes of reflux were analysed in detail: 82 (67\%) were synchronous with swallowing and 40 (33\%) asynchronous. Infants with trivial symptoms had gastro-oesophageal reflux synchronous with swallowing, whereas those with serious symptoms had slower acid clearance and asynchronous reflux. There were significant differences in lower oesophageal sphincter pressure and amplitude of oesophageal contractions between controls and patients with both gastro-oesophageal reflux and reflux oesophagitis. In reflux oesophagitis there was a decrease in lower oesophageal sphincter pressure and the contractions had a bizarre waveform suggesting a neuropathic process.

Gastro-oesophageal reflux is a common condition in infants and children that may be associated with significant morbidity due to an impaired energy intake, repeated pulmonary aspiration, and recurrent haemorrhage secondary to oesophagitis. ${ }^{1}$

Previously lower oesophageal sphincter activity has been regarded as crucial in the pathophysiology of gastro-oesophageal reflux, but this is probably an oversimplification. It is likely that mechanisms of gastro-oesophageal reflux involve the interplay of oesophageal motility, lower oesophageal sphincter activity, and gastric emptying. Disturbance of the mechanisms of clearance of gastric contents refluxed into the oesophagus may thus result from perturbation of the oesophageal motor apparatus. It has been suggested that spontaneous relaxation of the lower oesophageal sphincter in the absence of a preceding oesophageal peristaltic sequence ('inappropriate relaxation') is an important mechanism in gastro-oesophageal reflux, which may represent disturbed integrity of the oesophageal motor apparatus and be more important than reduced basal lower oesophageal sphincter pressure alone. ${ }^{2}$

In this study, motor function of the oesophagus, acid clearance, and the effects on oesophageal motility of exposing the oesophagus to endogenous acid, were assessed to evaluate the relationship between the disorders associated with gastro- oesophageal reflux described above and the motor mechanisms responsible for gastro-oesophageal reflux in patients with and without oesophagitis.

\section{Patients and methods}

Thirty three patients (age 3 months-14 years, median 2 years) with symptoms suggestive of gastrooesophageal reflux were investigated by barium meal, 24 hour oesophageal $\mathrm{pH}$ monitoring, oesophageal manometry, and endoscopy. Gastrooesophageal reflux was diagnosed by oesophageal pH monitoring and by the presence of reflux on barium meal examination. Nine patients (age 3 months -2 years) presented with feeding problems and failure to thrive; they did not have evidence of gastro-oesophageal reflux on $\mathrm{pH}$ studies or on barium meal and served as controls. Fifteen patients (age 3 months-4 years) had appreciable gastrooesophageal reflux on barium meal and $\mathrm{pH}$ study but did not have endoscopic evidence of oesophagitis; three of these patients had severe feeding problems and four recurrent respiratory problems. A further nine patients (age 1-14 years) were studied who had gastro-oesophageal reflux and appreciable oesophagitis on endoscopy. Four of the latter group have a major neurological condition: spastic quadriplegia or hydrocephalus. 
Twenty four hour oesophageal $\mathrm{pH}$ studies were performed using an antimony $\mathrm{pH}$ electrode (Synectics Medical) that was positioned in the oesophagus at the level of T10 using fluoroscopy, it was connected to a $\mathrm{pH}$ meter, the output of which was digitalised and stored for computer analysis on a solid state recorder. The electrode was calibrated at $\mathrm{pH} 1$ and $\mathrm{pH} 7$ before use. Appreciable gastrooesophageal reflux was present when oesophageal $\mathrm{pH}$ was less than 4 for more than $5 \%$ of a 24 hour period. Endoscopy was performed using an Olympus XP paediatric gastroscope.

Oesophageal motility was assessed by a continuous perfusion manometric technique. After a four hour fast and sedation with chlorpromazine (1 $\mathrm{mg} / \mathrm{kg}$ ) a triple lumen polyvinyl catheter (outside diameter $3 \mathrm{~mm}$ ), with side holes $4 \mathrm{~cm}$ apart and a distal Dent sleeve, ${ }^{3}$ was positioned fluoroscopically in the oesophagus with the Dent sleeve straddling the lower oesophageal sphincter. In vitro tests on the three transducers before use showed a pressure rise of $53.4 \mathrm{kPa} /$ second in response to sudden

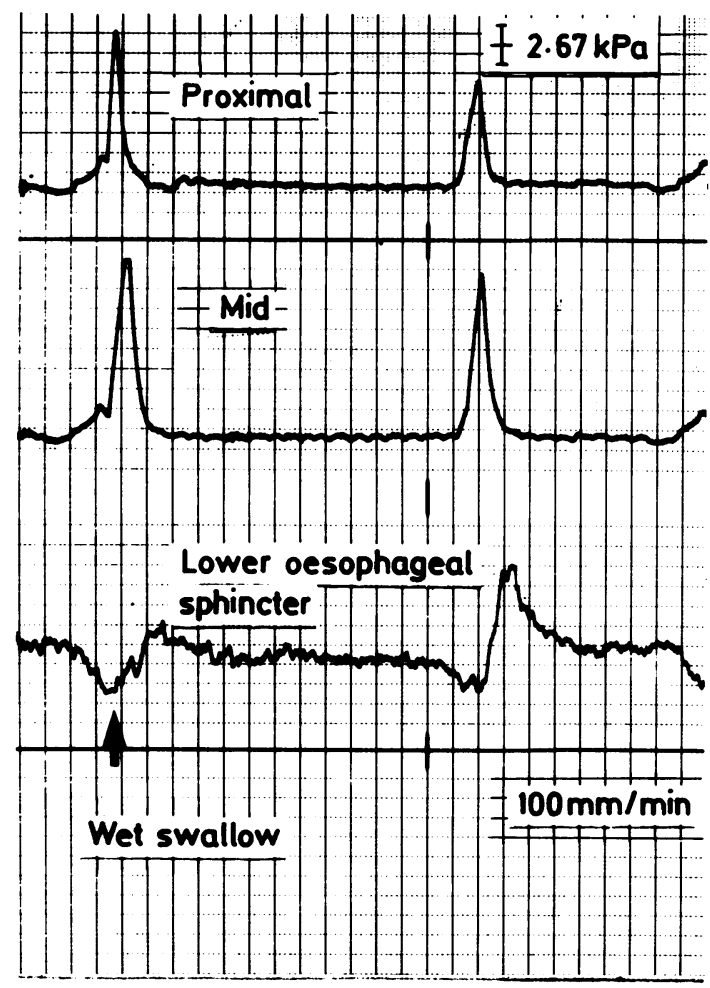

Fig 1 Normal oesophageal peristalsis recorded in a control patient from the proximal and midoesophageal body and from the lower oesophageal sphincter. occlusion of the three recording orifices. The catheter was constantly perfused $(1 \mathrm{ml} /$ minute $)$ with sterile water by a low compliance hydraulic capillary infusion system (Arndorfer Medical Specialties) and the proximal ends connected to three HewlettPackard pressure transducers (HP $1280 \mathrm{C}$ ) linked to an amplifier and chart recorder (HP 7754 B system). Pressure recordings were made from three levels: the proximal and midoesophageal body and the lower oesophageal sphincter, each separated by 4 cm (fig 1). The system was calibrated externally against a column of water and running speed was $100 \mathrm{~mm} /$ minute. Lower oesophageal sphincter pressure was measured taking atmospheric pressure as zero reference. Peristalsis induced by a wet swallow was recorded and the following motility variables were derived from the manometric recording: amplitude of contractions $(\mathrm{kPa})$, duration of contractions (seconds), and velocity of peristalsis ( $\mathrm{cm} /$ second). The configuration of the oesophageal pressure wave was assessed and the presence of multipeaked contractions noted.

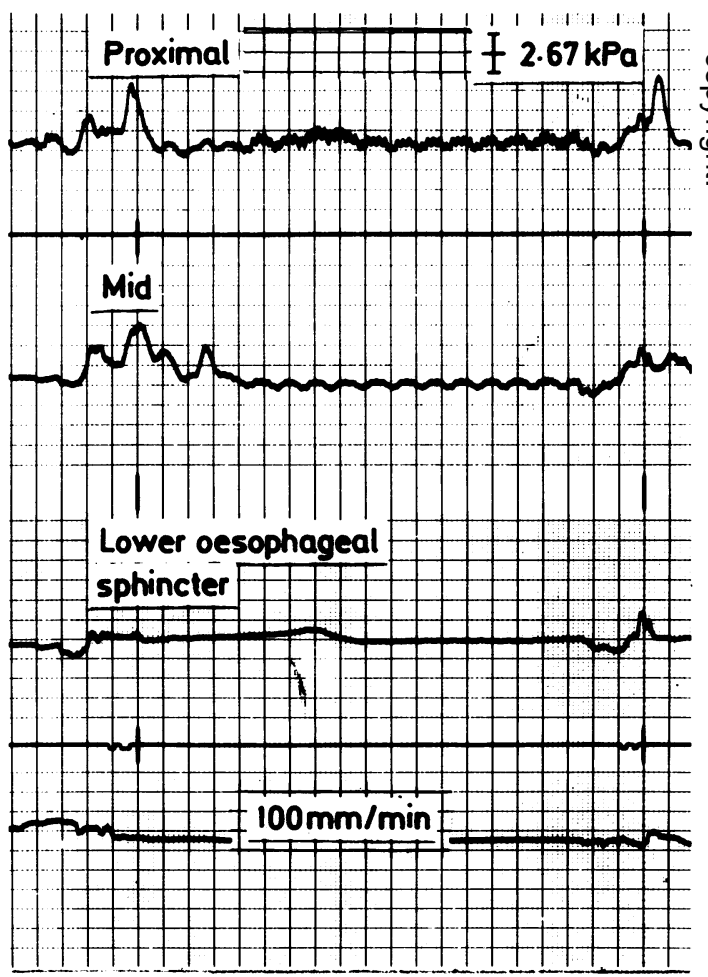

Fig 2 Wet swallow induced peristalsis in a child with reflux oesophagitis showing low amplitude multipeaked peristaltic waves. 
In the 15 patients with gastro-oesophageal reflux alone and the four patients with reflux oesophagitis, who had neurological abnormalities described above, combined oesophageal manometry and $\mathrm{pH}$ studies were performed. In these patients oesophageal manometry was performed as described above and, in addition, $\mathrm{pH}$ was recorded by attaching a small flexible $\mathrm{pH}$ electrode as used for 24 hour recordings $1 \mathrm{~cm}$ above the Dent sleeve of the manometric catheter and displaying the output of this electrode on the same polygraph as the manometric transducers. The $\mathrm{pH}$ electrode was calibrated in vitro at pH 1 and pH 7 before and after study. Gastrooesophageal reflux was defined as a sudden drop in intraoesophageal $\mathrm{pH}$ below 4 for more than 10 seconds.

Relaxation of the lower oesophageal sphincter was considered to be synchronous when it occurred after a peristaltic sequence induced by swallow and asynchronous when it occurred independently of a swallow. Peristaltic sequences were judged to be primary when preceded by swallowing and secondary when not preceded by swallowing.

All patients were studied in the supine position. Statistical analysis was performed on the data by Wilcoxon rank sum test for unpaired data. The study was approved by the standing committee on ethical practice of the Hospital for Sick Children and informed consent obtained from the parents of the children.

\section{Results}

\section{BODY OF THE OESOPHAGUS}

Configuration of the oesophageal pressure wave and patterns of motility

In all patients primary peristalsis was the most frequent motor pattern seen. An example of a primary peristaltic sequence induced by a wet swallow is shown in a control patient in fig 1 . In control patients and those with gastro-oesophageal reflux without oesophagitis the waveforms were sharp single peaks, which proceeded aborally down the oesophagus and at the lower oesophageal sphincter were preceded by clear relaxation of the sphincter. In the patients with oesophagitis the waveform of the contractions was bizarre: the contractions were widened and multipeaked of low amplitude (fig 2).

Secondary peristalsis in response to refluxed acid was seen both in patients with gastro-oesophageal reflux alone and in patients with reflux oesophagitis. As judged by acid clearance, however, in reflux oesophagitis it was often ineffective (see below).
Maximum amplitude of contractions $(\mathrm{kPa})$

There was a progressive decrease in the mean (SD) normal amplitude of contractions recorded from $9.60(2.13) \mathrm{kPa}$ in controls to $6.80(3.20) \mathrm{kPa}$ in patients with gastro-oesophageal reflux alone and $2.00(0.93) \mathrm{kPa}$ in patients with reflux oesophagitis. (When the controls were compared with each study group, $\mathrm{p}<0 \cdot 01$.)

\section{Duration of contractions (seconds)}

An increase in duration of contractions was observed in patients in both study groups compared with controls, but this was not significant. Mean (SD) values were $3.9(1.0)$ seconds in controls, 4.3 $(1 \cdot 1)$ seconds in patients with gastro-oesophageal reflux alone, and $6.8(2.5)$ seconds in patients with reflux oesophagitis.

\section{Velocity of peristalsis ( $\mathrm{cm} / \mathrm{second})$}

Mean (SD) velocity was similar in all patients: 2.9 $(1.6) \mathrm{cm} / \mathrm{second}$ in controls, $2.9(2 \cdot 1) \mathrm{cm} / \mathrm{second}$ in patients with gastro-oesophageal reflux alone, and

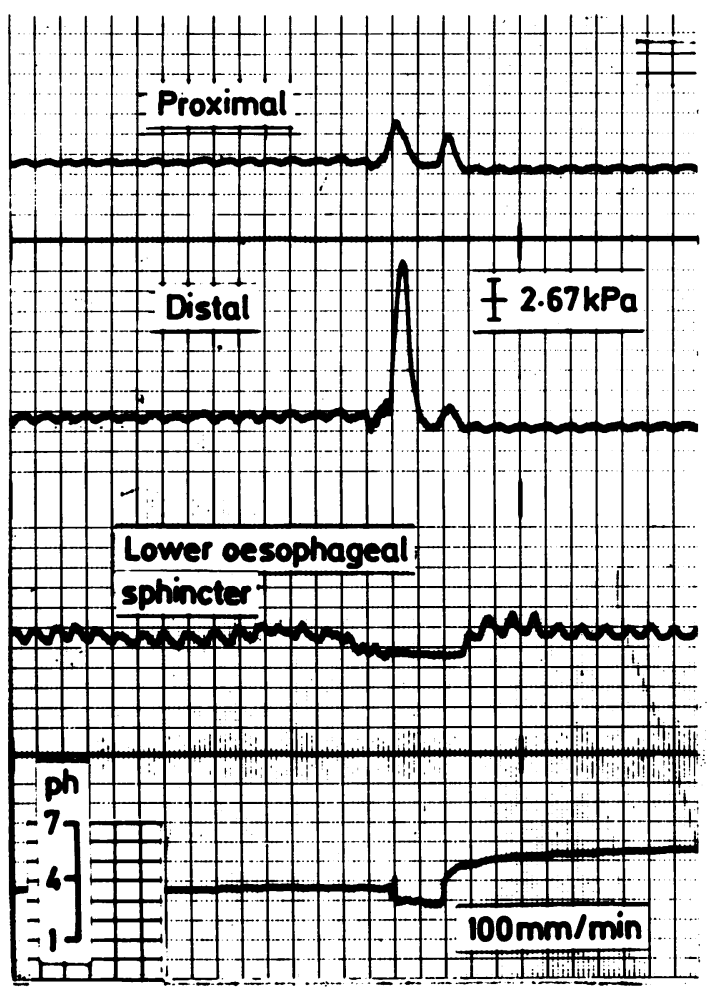

Fig 3 Gastro-oesophageal reflux occurring synchronously with swallowing. 
$2 \cdot 3(1 \cdot 1) \mathrm{cm} / \mathrm{second}$ in patients with reflux oesophagitis.

\section{LOWER OESOPHAGEAL SPHINCTER}

Lower oesophageal sphincter pressure $(k P a)$

Mean (SD) lower oesophageal sphincter pressure was similar in controls and patients with gastrooesophageal reflux alone $(2.93(0.53) \mathrm{kPa}$ compared with $2.93(1.6) \mathrm{kPa}$ ) but was significantly lower in patients with reflux oesophagitis $(1.87(0.93 \mathrm{kPa})$. (When the controls were compared with each study group, $\mathrm{p}<0 \cdot 01$.)

\section{Function of the lower oesophageal sphincter at time} of reflux

A total of 122 episodes of reflux were recorded and analysed: of these $82(67 \%)$ were synchronous with swallowing (as shown in fig 3 ) and $40(33 \%)$ were asynchronous (as shown in fig 4). Fifteen patients with gastro-oesophageal reflux alone were studied. Of 98 refluxes $76(78 \%)$ were synchronous and 22 $(22 \%)$ asynchronous in this group. The episodes of

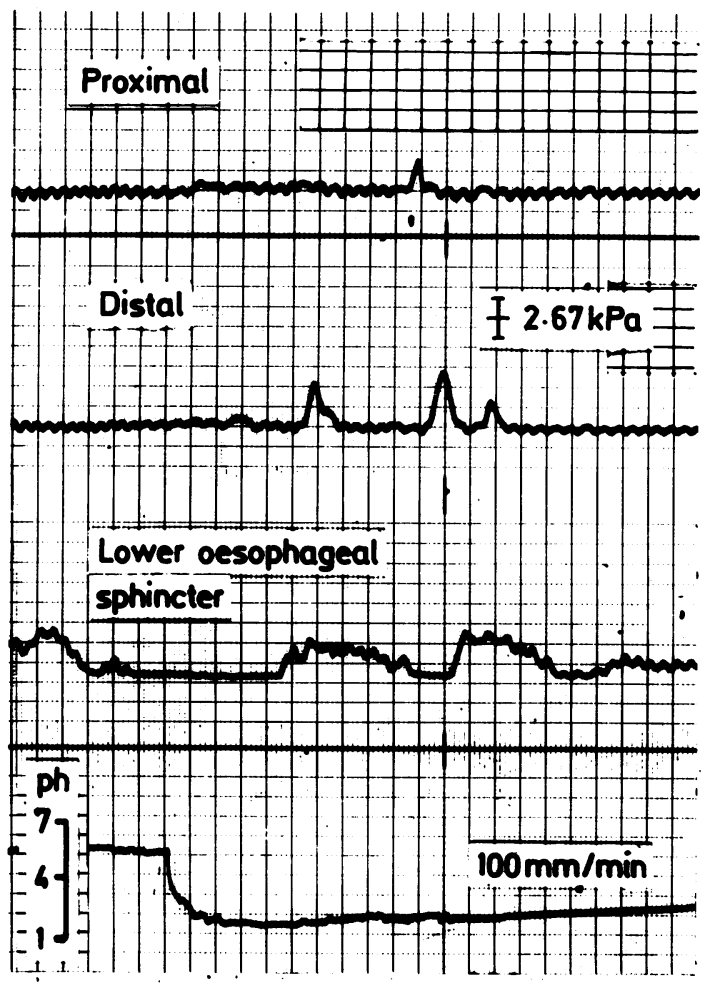

Fig 4 Gastro-oesophageal reflux occurring asynchronously with swallowing resulting in a prolonged reduction of oesophageal $\mathrm{pH}$ and ineffective secondary peristalsis. asynchronous reflux all occurred in seven patients who had either recurrent respiratory problems or severe feeding disorders. Mean (SD) clearance of refluxed acid was slower after asynchronous reflux than after synchronous reflux $(2.0(0.6)$ minutes compared with $0.6(0.1)$ minutes, $p<0.02)$. In the four patients with oesophagitis, who were also the patients with neurological problems, 24 episodes of reflux were studied: $18(75 \%)$ were asynchronous with swallowing and $6(25 \%)$ were synchronous. In this group mean (SD) acid clearance was even slower after asynchronous reflux (4.5 (0.9) minutes compared with $0 \cdot 6(0 \cdot 1)$ minutes, $\mathrm{p}<0 \cdot 01)$.

\section{Discussion}

The data from this study provide evidence of the nature of disordered motor activity in patients with gastro-oesophageal reflux and show that a variety of pathophysiological mechanisms may operate. Oesophageal motor function was more disturbed in those with both gastro-oesophageal reflux and reflux oesophagitis than gastro-oesophageal reflux alone. Amplitude of contractions and lower oesophageal sphincter pressures were significantly lower than controls and often bizarre in nature; these findings are similar to those noted in previous studies. ${ }^{45}$ In the patients with gastro-oesophageal reflux alone, however, we found no significant differences in oesophageal peristalsis and lower oesophageal sphincter pressure from the control patients.

Most $(78 \%)$ of the episodes of reflux that we observed in children with gastro-oesophageal reflux alone occurred synchronously with swallowing, whereas in patients with reflux oesophagitis most reflux episodes $(75 \%)$ were caused by relaxation of the lower oesophageal sphincter, which occurred asynchronously with swallowing. These findings are in accord with those of Baldi et al. ${ }^{6}$ Secondary peristalsis occurred very promptly in the patients in whom reflux occurred synchronously with swallowing (as shown in fig 3) resulting in a short lived episode of acid reflux, which was rapidly cleared. In those patients in whom reflux occurred asynchronously with swallowing acid clearance was much slower, being slowest in those with reflux oesophagitis, and secondary peristalsis was ineffective. In this latter group the $\mathrm{pH}$ only slowly increased suggesting that the mechanism of return to normal $\mathrm{pH}$ was not clearance but perhaps buffering by swallowed saliva. Thus secondary peristalsis associated with reflux synchronous with swallowing was more effective in clearing acid than secondary peristalsis that occurred after asynchronous reflux.

We also attempted to examine the effects of endogenous acid exposure on oesophageal motility. 
In those patients with reflux synchronous with swallowing refluxed acid was cleared promptly and induced a secondary peristaltic sequence, but those with asynchronous reflux had slower acid clearance and ineffective secondary peristalsis. Furthermore in the patients with reflux oesophagitis who had the slowest acid clearance bizarre multipeaked low amplitude waveforms were seen, and reflux asynchronous with swallowing occurred more often. It could be argued that both of these events result solely from the inflammatory response to prolonged acid exposure or that in addition there is an underlying motor abnormality, which results in less effective acid clearance. It is not completely clear from the present study which is the case as our study is cross sectional.

Our findings of reduced amplitude of smooth muscle contractions and bizarre multipeaked waveforms are similar to those previously reported. ${ }^{7} \mathrm{We}$ found in our previous studies in other regions of the gut that reduced amplitude of smooth muscle contractions is found in myopathic forms of intestinal pseudo-obstruction ${ }^{8}$; we also found bizarre waveforms with disturbed propagation in various neuropathic forms of intestinal pseudo-obstruction. ${ }^{9}$ It is possible that the disturbed motility observed in the patients with oesophagitis is simply as a consequence of the smooth muscle and oesophageal innervation operating in the environment of the inflammatory response to prolonged acid exposure. Some support for this hypothesis is provided by one study in which most children with oesophagitis and similar abnormalities to those reported here had their abnormal motor activity return to normal on resolution of the oesophagitis. ${ }^{10}$ Previous studies in adults, however, have shown that the abnormal motor events antedated the oesophagitis. ${ }^{11}$ In our patients with oesophagitis four suffered from neurological disease and these patients had the lowest lower oesophageal sphincter pressures and severely abnormal motility, and we have previously reported a series of children with brain stem tumours with severe reflux. ${ }^{12}$

Thus in patients with gastro-oesophageal reflux and more serious related disorders reflux was associated with severe disturbance of oesophageal motor activity in which not only was the contractile activity and control of the lower oesophageal sphincter impaired-that is, asynchronous or inappropriate relaxation of a poorly contracting sphincter occurred-but also peristaltic activity to remove refluxed acid was impaired and less effective. In patients with gastro-oesophageal reflux alone no such abnormalities could be detected and the cause of the reflux synchronous with swallowing that occurred was unclear, but it appeared to be very similar, but more frequent, to that which occurs physiologically on eating. ${ }^{15}$

The data from this study show that a variety of pathophysiological mechanisms may result in gastrooesophageal reflux. Decreased basal lower oesophageal sphincter pressure was an uncommon cause of gastro-oesophageal reflux and only occurred in reflux oesophagitis. In this group, however, reflux was more closely related to relaxation of the lower oesophageal sphincter, which occurred asynchronously with swallowing, rather than to reduced lower oesophageal sphincter pressure. Prolonged exposure of the oesophagus to acid resulting in inflammation may lead to a vicious circle being set up with further deterioration of oesophageal motor activity and even worse acid clearance.

Children with gastro-oesophageal reflux and trivial symptoms had gastro-oesophageal reflux synchronous with swallowing, and acid clearance occurred much faster than in those with serious symptoms. We suggest that reflux in those with gastro-oesophageal reflux alone is an exaggerated physiological response and may be treated by simple measures such as positioning and thickening of feeds, whereas in those with serious symptoms reflux may be related to intrinsic pathology of the oesophageal motor apparatus and require, in addition, the use of gastrointestinal prokinetic agents or, if these fail, surgery.

MJM is grateful to Janssen Pharmaceutical Ltd, for their generous financial support.

\section{References}

${ }^{1}$ Herbst JJ. Gastro-oesophageal reflux. J Pediatr 1981;98:859-70.

2 Dodds WJ, Dent J, Hogan WJ, et al. Mechanisms of gastrooesophageal reflux in patients with reflux oesophagitis. $N$ Engl $J$ Med 1982;307:1547-52.

3 Dent J. A new technique for continuous sphincter pressure measurement. Gastroenterology 1976;71:263-7.

4 Hillemeier AC, Grill BB, McCallum R, Gryboski J. Esophageal and gastric motor abnormalities in gastro-esophageal reflux during infancy. Gastroenterolgy 1983;84:741-6.

5 Cucchiara S, Staiano A, DiLorenzo C, et al. Esophageal motor abnormalities in children with gastro-esophageal reflux and peptic esophagitis. J Pediatr 1986;108:907-10.

6 Baldi F, Ferrarini F, Balestra R, et al. Oesophageal motor events at the occurrence of acid reflux and during endogenous acid exposure in healthy subjects and in patients with oesophagitis. Gut 1985;26:336-41.

${ }^{7}$ Loo FD, Dodds WJ, Soergel KH, Arndorfer RC, Helm JF, Hogan WJ. Multipeaked esophageal peristalitic pressure waves in patients with diabetic neuropathy. Gastroenterology 1985;88: 485-91.

${ }^{8}$ Milla PJ, Lake BD, Spitz L, Nixon HH, Harries JT, Fenton TR. Chronic idiopathic intestinal pseudo-obstruction in infancy: a smooth muscle disease. In: Labo G, Bortolotti M, eds. Gastrointestinal motility. Verona: Cortina International, 1983: 127-31. 
9 Wozniak ER, Fenton TR, Milla PJ. Fasting small intestine motor activity in chronic idiopathic pseudo-obstruction. Pediatr Res 1984;18:1060.

10 Cucchiara S, Staiano A, Capozzi C, Di Lorenzo C, Boccieri A, Auricchio S. Cisapride for gastro-oesophageal reflux and peptic oesophagitis. Arch Dis Child 1987;62:454-7.

11 Kahrilas PJ, Dodds WJ, Hogan WJ, Kern M, Arndorfer RC, Reece A. Esophageal peristaltic dysfunction in peptic esophagitis. Gastroenterology 1987;91:897-904.

12 Mahony MJ, Kennedy JD, Leaf A, Matthew DJ, Milla PJ. Brain stem glioma presenting as gastro-oesophageal reflux. Arch Dis Child 1987;62:731-3.

13 Dent J, Dodds WJ, Friedman RH, et al. Mechanisms of gastrooesophageal reflux in recumbent asymptomatic human subjects. J Clin Invest 1980;65:256-67.

Correspondence to Dr MJ Mahony, Department of Paediatrics, St James's University Hospital, Beckett Street, Leeds LS9 7TF.

Accepted 25 April 1988 